\title{
Art rupestre post-glaciaire de Cerdagne
}

\section{Pierre Campmajo}

URL : http://journals.openedition.org/adlfi/12059

ISSN : 2114-0502

Éditeur

Ministère de la culture

Référence électronique

Pierre Campmajo, «Art rupestre post-glaciaire de Cerdagne », ADLFI. Archéologie de la France-

Informations [En ligne], Languedoc-Roussillon, mis en ligne le 01 mars 2004, consulté le 20 avril 2019. URL : http://journals.openedition.org/adlfi/12059

Ce document a été généré automatiquement le 20 avril 2019.

(c) Ministère de la Culture et de la Communication, CNRS 


\title{
Art rupestre post-glaciaire de Cerdagne
}

\author{
Pierre Campmajo
}

Date de l'opération : 1992 - 1997 (PI)

Inventeur(s) : Campmajo Pierre

Menée dans le cadre de l'Inventaire national des sites (programme AH), cette recherche sur l'art rupestre post-glaciaire de Cerdagne débute avec l'arrivée des Ibères dans cette région, dès la fin du $\mathrm{III}^{\mathrm{e}}$ s. av. J.C. Nous étudions ici une tranche de l'archéologie qui se rapproche de l'histoire et ne prenons pas en compte les cupules et autres symboles se rapportant à l'art dolménique. La relative jeunesse des gravures n'enlève rien à leur intérêt et montre comment, dès cette époque, la Cerdagne récupère les rites religieux de cette culture ibère et toute la symbolique qui s'y rapporte. Mais pour comprendre ce phénomène, un bref rappel des cultures qui précèdent l'arrivée de Ibères en Cerdagne nous semble nécessaire.

Nous voici bien après les premières traces d'occupations néolithiques. À l'âge du Bronze (entre $1800 \mathrm{av}$. J.-C et $1000 \mathrm{av}$. J.-C.), la Cerdagne connaît une très grande expansion démographique et les sites se comptent par dizaines. Dès l'an 1000 av. J.C., la civilisation dite " des Champs d'urnes » diffuse lentement de la côte catalane vers les hautes terres; c'est une période culturelle et technique importante. La Cerdagne assimile rapidement ces influences, les fondant à ses propres productions pour donner naissance à une culture locale que les archéologues appellent « céramique à décor cerdan ».

Les sept siècles précédant l'avènement du Christ verront cette culture se propager rapidement dans les contrées voisines (piémonts nord et sud des Pyrénées et Pyrénées centrales). Les auteurs antiques désignent du nom de Keretes, ces peuples puissants, belliqueux et très indépendants. C'est peut-être pour ces raisons que les Ibères et les Romains ne s'y aventureront que très tardivement, à la fin du $\mathrm{III}^{\mathrm{e}} \mathrm{s}$. pour les Ibères et en 30 av. J.-C., pour les Romains. 


\section{La culture ibère en Cerdagne}

La culture ibère naît au sud de la chaîne des Pyrénées, près de la Méditerranée, dans l'espace de ce qui deviendra plus tard la Catalogne. Elle est issue de la proximité de la mer qui engendre, précocement (dès le $\mathrm{VII}^{\mathrm{e}} \mathrm{s}$. av. J.-C.) des contacts commerciaux réguliers avec les Phéniciens d'abord puis les Grecs et les Puniques ensuite. Depuis longtemps, ces peuples orientaux maîtrisent l'écriture, inconnue dans nos contrées. Rapidement, les Ibères, d'abord par obligation commerciale puis pour des raisons religieuses, adoptent et remodèlent ces alphabets venus de la mer, pour créer une écriture nouvelle connue sous le nom d'écritureibère (malgré les recherches développées sur le sujet, la langue que transcrit cette écriture reste encore inconnue). Cette écriture et la culture ibère connaîtront un essor exceptionnel jusqu'à la fin du III ${ }^{\mathrm{e}}$ s. av. J.-C.

En 218 avant J.C., les Romains débarquent à Empuries pour intervenir militairement en Espagne lors des guerres puniques qui empoisonnent les rapports entre Rome et Carthage. Ces conflits sont loin de la Cerdagne, alors en quoi peuvent-ils l'intéresser ? Au début, Romains et Ibères entretiennent de bons rapports mais l'Espagne est riche: les gisements d'or, d'argent et cuivre foisonnent, l'agriculture et l'élevage sont florissants. Rome a de gros besoins financiers pour maintenir son empire, aussi, rapidement, les Romains s'enfoncent dans les terres pour exploiter ces richesses. Cela ne se fait pas sans heurt, à tel point que dans les années 186-183 av. J.-C., les Ibères se rebellent. Leur révolte est durement réprimée par le consul Caton.

Si les populations cerdanes du second âge du Fer (500-100 av. J.-C.) avaient certainement des contacts avec leurs voisins du sud, ne serait-ce qu'à l'occasion de rencontres pastorales sur les estives et d'un petit commerce, la culture ibère, si forte au sud des Pyrénées n'avait pas jusqu'alors, influencé les habitants des montagnes. L'écriture y était inconnue et le mobilier ibère totalement absent. Or, c'est au début $d u \mathrm{II}^{\mathrm{e}} \mathrm{s}$. que les premières céramiques importées de la côte catalane apparaissent sur le site de Llo. C'est du début $\mathrm{du} \mathrm{II}^{\mathrm{e}} \mathrm{s}$. aussi que sont datées les écritures ibères trouvées sur les roches de Cerdagne. Comment ne pas relier cette irruption soudaine, cette acculturation tardive à la répression de Caton? Les Ibères sont obligés de collaborer, mais une minorité s'est enfuie vers les montagnes, zone refuge par excellence. Paradoxe : alors qu'au sud des Pyrénées, les Romains latinisent les peuples ibères au point que leur langue a pratiquement disparu à la fin du II $\mathrm{e}$ s. av. J.-C., la Cerdagne devient un conservatoire de l'écriture et peut-être aussi de la langue, et cela pour longtemps encore.

Les Romains n'envahiront la Cerdagne fondant la ville de Llivia que vers 40-30 av. J.-C. Restée à l'écart des conflits pendant près de deux siècles, la haute vallée du Sègre n'intègre donc que tardivement l'espace contrôlé par Rome.

\section{Les écritures et les gravures rupestres de Cerdagne}

Les recherches sur les gravures ont permis de constituer un corpus de gravures et d'écriture très important. Aujourd'hui, ce sont plus de sept cent cinquante signes en lettres ibères qui ont été recensés en Cerdagne. Ces écritures sont accompagnées de dessins et de signes symboliques : arboriforme (en forme d'arbre), arciforme (en forme d'arc), arbalétiforme (en forme d'arbalète), scaliforme (en forme d'échelle), pentacles, croix, nœuds tressés sur le modèle de la svastika indo-européenne, scènes de chasse, etc. 
Certains de ces signes sont donc contemporains des écritures ibères mais d'autres datent du Moyen Âge : ils accompagnent des représentations de guerriers en armes, des chevaux, etc. D'autres enfin, plus récents, ont été tracés par des bergers ou des carriers, qui ont souvent eu la bonne idée de graver leur nom et d'y apposer une date. Aujourd'hui encore, le simple promeneur se plait à graver la roche, risquant par là, sans le savoir, de détruire des gravures plus anciennes, souvent difficiles à voir. Ainsi, les recherches en cours, malgré une attribution chronologique délicate, permettent-elles souvent de dater les dessins, au moins par grandes périodes. Mais écritures et dessins, que signifient-ils?

L'étude que nous menons sur ce patrimoine, montre de façon très claire que la majorité de ces lieux sont des sanctuaires, pour la plupart dédiés aux cultes anciens des sources, des arbres, des roches. Les signes symboliques qui se répètent dans le temps, montrent que ces vieilles croyances ont largement perduré, bien après l'avènement du christianisme. Bien des légendes en témoignent, qui sont arrivées jusqu'à nous : haches en pierre polie que l'on posait sur le bord des fenêtres pour préserver la maison de la foudre, tuiles retournées ou masques en terre cuite fixés aux faîtes des toits étaient censés éloigner les sorcières et les mauvais esprits. Les vieilles ferrures des poignées de porte de nombre de fermes étaient décorées de signes prophylactiques en forme de croix, coq et autres symboles qui protégeaient hommes et troupeaux. Toutes ces croyances qui sont le reflet des cultes anciens, nourrissent l'art rupestre.

Le corpus des roches gravées de Cerdagne étant important et d'intérêts divers, nous proposons quelques exemples parmi les ensembles les plus significatifs.

\section{Osséja}

\section{Osséja-Genèvri - Zone 2 - Roche 5 n56}

\section{Description}

Cavalier à pied, menant son cheval par la bride. De la main gauche, il tient une longue lance, sa coiffure est un casque rond et pointu posé sur le camail lui couvrant les épaules. Il porte, fixée au niveau de la ceinture côté gauche, une longue épée à pommeau circulaire. Le cheval, bien dessiné, porte une selle à bâtes avant et arrière. Elle est maintenue par une sous-ventrière et une croupière [ (Fig. $\mathrm{n}^{\circ} 1$ : Osséja - Zone 2 - Roche 5 , $\mathrm{n}^{\circ} 56$ - Cavalier à pied menant son cheval par la bride. Fin XIIe s. - début XIIIe s.) et (Fig. $\mathrm{n}$ ${ }^{\circ} 2$ : Osséja - Zone 2 - Roche $5, n^{\circ} 56$ - Détail du cavalier à pied menant son cheval par la bride. Fin XIIe s. - début XIIIe s.)]. Un graffite illisible oblitère le dessin.

\section{Observation}

Cette représentation est, pour la chronologie des gravures, d'une grande importance, car les nombreux détails vestimentaires, l'armement et le harnachement du cheval sont parfaitement datables. Pour analyser ces éléments, nous nous sommes servis de la description de la tapisserie de Bayeux (datée de 1066) et de l'Encyclopédie médiévale de Viollet-le-Duc. Ces deux ouvrages décrivent parfaitement les tenues vestimentaires militaires du Moyen Âge. 


\section{Le casque}

Sur la tapisserie de Bayeux, le casque des combattants des deux armées s'affrontant est conique avec présence du nasal. Ce nasal perdurera jusqu'au début du XIII ${ }^{\mathrm{e}} \mathrm{s}$. Le casque conique, sous la forme la plus simple comme celle de notre dessin, durera jusqu'au début du XIV ${ }^{e}$ s., mais son apogée se situe aux XI ${ }^{e}$ s. et XII $s$.

\section{Le camail}

Est une coiffure de tissu ou de mailles d'acier qui protégeait le crâne et les épaules. Souvent rembourré, il permettait de supporter le lourd casque de fer, mais avait aussi pour effet d'amortir les coups portés par l'adversaire. Le camail se portait sur le haubert et il est très rare qu'ils soient dissociés avant la fin du XIII ${ }^{e} s$. ou le début du XIV ${ }^{e} s$. Dès cette époque, il est parfois ouvert sur le devant pour laisser une plus grande mobilité au visage. C'est peut-être le cas sur notre dessin. L'armement de notre cavalier se limite à une lance et une épée. La position de l'épée portée très oblique n'est pas le simple fait du hasard ; cela est dû à sa longueur, qui sur notre représentation dépasse largement le mètre. Or, on sait que les épées de cette taille n'apparaissent qu'à la fin du XII ${ }^{e} \mathrm{~s}$. ou au tout début du XIII $\mathrm{s}$. Ces épées, plus lourdes, nécessitent un baudrier particulier qui n'est pas représenté sur notre dessin.

\section{La lance}

Proportionnellement, comparée à la taille du cavalier, la lance de notre cavalier devait mesurer environ $3 \mathrm{~m}$. Il s'agit donc d'une lance courte que les cavaliers utilisent dès le $\mathrm{XI}^{\mathrm{e}}$ $\mathrm{s}$. Au XIV e s. et au XV es., elle s'allonge considérablement pour atteindre les $5 \mathrm{~m}$. Sur le type court, le fer de lance a souvent une forme losangée identique à celle qui figure sur notre dessin.

\section{Cheval et harnachement}

\section{Le cheval}

Le graphisme du cheval lui-même appelle peu de commentaire si ce n'est sa belle taille et la longueur un peu démesurée de son cou. Les pattes ne sont que partiellement dessinées.

\section{La selle}

La selle à bâtes mérite un commentaire, bien qu'elle ne soit pas un élément chronologique fiable du fait de son invention très ancienne. Les bâtes apparaissent en effet dès l'utilisation des lances pour charger, au moins à partir du VIII $\mathrm{s}$. La cavalerie de Charlemagne utilisait déjà ce type de selle, la seule différence est que les bâtes étaient plus basses. 
La lance longue se développe vers la fin du $\mathrm{XII}^{\mathrm{e}} \mathrm{s}$. À partir de cette époque, les bâtes deviennent plus hautes, ceci afin de bien caler le cavalier dans la selle. Sur notre dessin, la selle est positionnée trop en arrière. Ce type de selle se place plus en avant sur le garrot du cheval, car le cavalier charge debout sur les étriers, le corps penché en avant ; ainsi porté, le coup de lance a une force très supérieure à tout autre mode d'utilisation.

La forme des bâtes représentée à Osséja nous fait penser qu'il s'agit d'un modèle assez fermé ceinturant bien le cavalier au niveau des hanches. C'est au cours du XIII ${ }^{\mathrm{e}}$ s. que ce modèle se développe le plus.

La croupière qui enveloppe la croupe du cheval se développe, elle aussi, surtout au XIII ${ }^{e} \mathrm{~s}$., mais certaines représentations la font remonter à l'époque byzantine au VIII $\mathrm{s}$.

$\mathrm{Si}$ on analyse ensemble toute la documentation décrite ci-dessus, (tenue vestimentaire du cavalier, armement et harnachement), on arrive à un ensemble de données offrant une fourchette chronologique assez large. Mais elle se resserre dès que l'on touche à des points de détails. Ainsi, le casque rond et pointu avec ou sans nasal s'utilise entre le $\mathrm{XI}^{\mathrm{e}} \mathrm{s}$. et le XIV ${ }^{e} s$. Le camail ouvert est très en vogue au XIII ${ }^{e} s$. Les longues épées ne sont courantes qu'à la fin du XII ${ }^{\mathrm{e}} \mathrm{s}$. et au début XIII ${ }^{\mathrm{e}} \mathrm{s}$. La lance courte est largement utilisée au $\mathrm{XI}^{\mathrm{e}}$ s. comme l'atteste à plusieurs reprises la Tapisserie de Bayeux. Bâtes et croupières sont connues dès le $\mathrm{VIII}^{\mathrm{e}} \mathrm{s}$., mais leur développement maximum se situe au XII ${ }^{\mathrm{e}}$ s. et au XIII ${ }^{\mathrm{e}} \mathrm{s}$.

L'utilisation maximale de tous les éléments figurant sur notre dessin tournent tous, plus au moins, vers la fin du XII ${ }^{e}$ s. - début XIII ${ }^{e}$ s. C'est la date que nous proposons pour notre cavalier.

\section{Date présumée}

Moyen Âge.

\section{Osséja-Genévri - Zone 2 - Roche $1 n^{\circ} 4$}

\section{Description}

Deux sujets armés, habillés d'une longue tunique et entourés d'oiseaux [ (Fig. n³ : Osséja - Zone 2 - Roche $1, \mathrm{n}^{\circ} 4$ - Groupe de chasseurs ou de guerriers en armes. Époque médiévale) et (Fig. $\mathrm{n}^{\circ} 4$ : Osséja - Zone 2 - Roche $1 \mathrm{n}^{\circ} 4$ - Détail du panneau représentant un sujet anthropomorphe en arme, surmonté d'un oiseau)].

Le sujet de gauche tient dans sa main droite une épée à large lame, décorée d'un zigzag. Cette épée recoupe un arbalétiforme à jambe latérale droite. Une lance oblitère le bras gauche du sujet. Le bras droit est replié et vient s'appuyer sur la hanche. Les mains sont ramifiées. Les pieds sont triangulaires tournés vers la droite dans un mouvement de marche. Le sujet est habillé d'une longue tunique hachurée, les hachures sont dans le sens gauche. Un pli dans le vêtement est marqué au niveau des pieds par une opposition des traits obliques tournés à droite. La tête du sujet est ronde et doublée d'un deuxième trait figurant les cheveux ou une coiffure. Le cou est long, un X est dessiné au centre. À droite du sujet, un oiseau à longues pattes croisées a la tête surmontée de deux petits traits obliques comme sur une tête d'aigrette. 
Un grand bouclier est divisé en quatre panneaux, disposés en métopes, décorés de lignes verticales et horizontales. À droite de ce bouclier, une figure ovoïde pourrait, elle aussi, représenter un bouclier. Le décor est fait de lignes obliques tournées vers le haut s'articulant autour d'un axe central très long qui se termine par une ramification à quatre branches. Ce détail laisse supposer que la figuration du bouclier doit être abandonnée au profit d'un motif, peut-être anthropomorphe.

À droite, le second sujet a le bras droit levé ; sa main ramifiée tient une très longue lance qui part du deuxième oiseau jusqu'à la pointe de la lance. À ce niveau, la roche est cassée et la frise supérieure est indépendante de la scène. À droite du panneau, il y a encore deux lances dont les pointes sont coupées. L'oiseau du bas semble avoir un long plumage, l'ensemble des autres traits est plus difficile à interpréter.

La frise supérieure peut être lue de gauche à droite : croix coupée par des diagonales, puis carré avec médianes et diagonales tracées. À droite, croix latine où les extrémités de la barre horizontale sont potencées, suivie de trois signes $\mathrm{X}$.

\section{Observation}

La signification de cette scène est complexe. S'agit-il d'une scène guerrière comme le laisse supposer les nombreuses armes et le bouclier présent sur le panneau? Ce n'est pas sûr.

Quelle signification faut-il accorder aux oiseaux ? Est-ce une scène de chasse ? À notre avis, l'élément le plus important est le motif ovoïde à décor de hachures obliques dont l'axe central se termine par une ramification. Ces ramifications sont importantes quand nous avons des représentations d'arbres ou de palmettes ramifiés au niveau de l'axe central. L'oiseau a, lui aussi, sa mythologie dans les croyances populaires.

La datation de cette gravure peut, par les nombreux recoupements que nous avons pu faire, avoir son importance. Nous avons en effet constaté statistiquement que les sujets habillés et armés étaient très probablement d'époque médiévale. Les pieds triangulaires nous semblent être un fossile-directeur de cette période. Les longues lances appartiennent plutôt à des cavaliers qu'à des fantassins. Tous ces éléments nous font pencher pour une datation d'époque médiévale sans plus de précision.

\section{Date présumée}

Époque médiévale.

\section{Osséja-Genévri - Zone 2 - Roche $4 n^{\circ} 19$}

Groupe d'anthropomorphes aux formes diverses (Fig. n8 8 : Osséja - Zone 2 - Roche $4 \mathrm{n}$

- 19 - Groupe d'anthropomorphes aux formes diverses. Époque ibère).

\section{Situation}

Légèrement en dessous et au sud de la Roche $3 n^{\circ}$ 26, altitude 1420 m. 


\section{Support}

Schiste

\section{Technique}

Linéaire

\section{Description}

Scène à plusieurs anthropomorphes. Lecture de gauche à droite : dix lignes parallèles verticales. Dessous, frise de deux traits parallèles obliques remplie de deux lignes parallèles, un X, deux lignes parallèles ; un trait oblitère le dessin. À droite, deux lignes parallèles horizontales coupées d'une ligne oblique.

\section{Anthropomorphe filiforme}

Le dessin s'articule autour d'un axe central ; à son extrémité supérieure, la tête en forme de poire inversée porte trois points, deux figurant les yeux, le troisième, le nez ou la bouche. Les bras élevés en orant finissent chacun par cinq ramifications pectiniformes. Les membres inférieurs sont marqués par duc lignes droites dont celle de droite ramifiée à son extrémité inférieure. La ligne représentant le corps dépasse l'intersection des membres inférieurs. Y-a-t-il eu volonté de représenter le sexe ? C'est possible.

Dessous, la ligne courbe terminée à son extrémité supérieure par une pointe bifide. À droite, à côté du premier anthropomorphe, il y a un second anthropomorphe filiforme. La tête est représentée par un demi-cercle ; le corps est une ligne verticale avec les bras figurés en orant partant vers le haut ; le bras droit se finit par cinq ramifications pectiniformes. Les jambes sont rectilignes. La gauche s'achève par cinq ramifications pectiformes ; le sexe n'est pas représenté.

À droite, trois lignes verticales parallèles légèrement obliques ; celle de droite finit par un croisillon. À droite, un arciforme-anthropomorphe composé d'un arc de cercle positif ; la corde est coupée par quatre courtes lignes verticales. À droite, motif en grille à deux jambes. Au-dessus, anthropomorphe filiforme. La tête vide de tout organe, est ronde et repose sur un axe vertical. Du corps partent les membres inférieurs représentés par deux lignes courbes. La droite est plus longue que la gauche. Les bras en orant sont un arc de cercle divisé par la ligne du corps. Le bras droit se termine par cinq ramifications pectiformes tournées vers le bas.

\section{Observation}

Nous avons une véritable scène composée en frise regroupant nombreux éléments symboliques souvent figurés sur les représentations à fort contexte ibère. L'élément en grille et les traits parallèles, arciformes dans le contexte de la scène, doivent être interprétés comme anthropomorphe. On pourrait se poser la même question pour les 
trois lignes parallèles se trouvant au milieu de la scène. Enfin, la petite frise de gauche est un élément très souvent évoqué en contexte ibère. Les deux derniers dessins, lignes parallèles horizontales barrées et la ligne courbe bifide restent des éléments mystérieux. Cette scène symbolique narre un événement dont nous ne sommes pas prêts de trouver la clef.

\section{Date présumée}

Ibère, sous réserve.

\section{Osséja - zone 1 - Roche $3 n^{\circ} 1$}

\section{Description}

Scène composée d'éléments figuratifs, anthropomorphes masculins ou féminins, de chevaux et d'éléments symboliques : scalariforme, réticulé, spirale, traits radiants et zigzag. Quelques traits " parasites » oblitèrent la scène (Fig. $n^{\circ} 1$ : Osséja - Zone 2 - Roche $5, n^{\circ} 56$ - Cavalier à pied menant son cheval par la bride. Fin XIIe s. - début XIIIe s.8).

\section{Observation}

Outre les éléments symboliques, l'homme et les chevaux sont à observer plus particulièrement.

L'homme semble être coiffé d'une " tiare " tronconique. Son oreille gauche porte un pendant. Cet élément est suffisamment rare en Cerdagne pour être signalé. La tenue vestimentaire est faite d'une sorte de jupe aux motifs striés en métopes qui se retrouvent fréquemment sur les gravures. C'est certainement un marqueur chronologique qu'il faudra bien observer. Des traits filiformes représentent la main gauche du sujet. Le cheval supérieur, mis à part son archaïsme et l'extrémité de ses pattes, n'a rien de spécialement remarquable si ce n'est la rusticité du dessin. Le cheval du dessus, certainement tracé par la même main, est plus intéressant. Bien que la tête soit absente, la crinière est marquée et il ne fait pas de doute que l'on soit en présence d'un cheval. Sa robe est étonnante par sa composition, faite d'une succession de quarts de cercles concentriques. La position des pattes croisées peut laisser supposer que l'animal a été dessiné en train de marcher.

Nous retrouvons ce même mouvement à Osséja - Zone 2 - Roche $21 \mathrm{n}^{\circ}$ 12. Les dessins sont tellement ressemblants qu'on peut se demander s'ils n'ont pas été tracés par la même personne.

\section{Date présumée}

Moyen Âge. 


\section{Osséja-Corbera Gassiot - zone 6 - Roche $2 n^{\circ} 1$}

\section{Description}

Quatre lignes de graffiti ibères superposées (Fig. $\mathrm{n}^{\circ} 5$ : Osséja-Corbera - Zone 6 - Roche $2 \mathrm{n}$

- 1 - Quatre lignes d'écriture ibère. Époque ibère - 200 à 100 av. J.-C.).

\section{Ligne 1}

Dix lettres parfaitement tracées.

\section{Ligne 2}

Elle peut être divisée en quatre groupes de lettres

Groupe $1:$ six lettres ;

Groupe 2 : quatre lettres;

Groupe 3 : quatre lettres;

Groupe 4 : dix lettres légèrement plus grandes.

\section{Ligne 3}

Elle compte deux groupes de lettres.

Groupe 1 : treize lettres d'une hauteur moyenne de $12 \mathrm{~cm}$;

Groupe 2 : sept lettres d'une hauteur moyenne variant entre $0,6 \mathrm{~cm}$ et $0,9 \mathrm{~cm}$.

\section{Ligne 4}

Elle est composée de 2 groupes de lettres de hauteurs différentes.

Groupe 1 : sept lettres d'une hauteur de 7 centimètres;

Groupe 2 : sept lettres d'une hauteur moyenne de 17 centimètres.

\section{Observation}

Toutes ces lignes semblent écrites de la même main (?).

\section{Date présumée}

Ibère. 


\section{Osséja-Corbera Roche Rendut - Roche $1 n^{\circ} 1$ Description}

Trois lignes de lettres ibères superposées (Fig. $\mathrm{n}^{\circ} 6$ : Osséja-Corbera - Zone 6 - Roche $1 \mathrm{n}^{\circ} 1$ - Ecriture ibère. Époque ibère - 200 à 100 avant J.-C.).

\section{Observation et analyse (J. Untermann)}

On reconnait trois lignes, dont la première montre un ductus différent, tandis que les deuxième et troisième lignes sont dues à la seule main (Fig. n ${ }^{\circ} 1$ : Osséja - Zone 2 - Roche 5 , $\mathrm{n}^{\circ} 56$ - Cavalier à pied menant son cheval par la bride. Fin XIIe s. - début XIIIe s.9).

\section{Date présumée}

Ibère

\section{Osséja-Carrlère de la Cabanette - Roche $1 \mathrm{n}^{\circ} 1$}

\section{Description}

Guerrier armé associé à un faisceau de lances (Fig. $n^{\circ} 7$ : Osséja-Carrière de la Cabanette Roche $1 \mathrm{n}^{\circ} 1$ - Guerrier armé associé à un faisceau de lances. Moyen Âge, sous réserve).

La tête repose sur un cou représenté par quatre lignes verticales obliques. Le corps, dans sa partie supérieure, a une forme triangulaire. Deux lignes droites verticales portent les pointes du triangle, lignes droites verticales formant d'un seul trait le contour du corps et des jambes. À l'extrémité inférieure de ces lignes, des traits parallèles obliques représentent les pieds, quatre traits pour la jambe droite et trois pour la gauche. Ils sont tournés vers la gauche du panneau. Deux triangles, traversant les lignes verticales, figurent le haut des jambes. Le corps est rempli de lignes obliques formant des croisillons. Le sujet est, semble-t-il, de sexe masculin comme le confirme un triangle allongé strié. Curieusement, le bras droit part près du cou. La main est filiforme et ramifiée. Elle tient une longue épée à pommeau arrondi. La lame est partagée en deux parties égales par une longue ligne horizontale qui part de la pointe jusqu'au cinquième de la longueur environ. Une série de courtes lignes obliques coupe régulièrement cette ligne. La partie figurant la poignée n'est pas décorée.

Le bras gauche part du coin de l'épaule (position normale), il est coudé et se termine par une main également ramifiée tenant une lance au fer démesuré.

À gauche du sujet, il y a deux signes en forme d'étoile fait d'axes radiants. À droite du guerrier, on voit une horizontale recoupant un faisceau de quatre lances verticales. 


\section{Observation}

Ce dessin se trouve sur la partie supérieure de la roche, à $3 \mathrm{~m}$ environ de la Roche $1 \mathrm{n}^{\circ} 4$ représentant un graffite ibère. Le graphisme du sujet, l'épée à pommeau arrondi et les lances plaident pour une datation médiévale. Restent les deux étoiles qui peuvent être lues comme signe ibère $\mathrm{j}=\mathrm{bo}$, ou bien comme symbole solaire à axes radiants. Ces mêmes signes se retrouvent sur la Roche $5 \mathrm{n}^{\circ} 7 \mathrm{du}$ site de Cabanette dans une ambiance ibère.

\section{Date présumée}

Époque médiévale, sous réserve.

\section{Err}

\section{ERR-Roc de Carbanet - Roche $4 n^{\circ} 2$}

\section{Description}

Panneau avec une série de dessins et de symboles que l'on retrouve fréquemment dans les roches d'ambiance ibérique [ (Fig. $n^{\circ} 9$ : Err-Carbanet - Roche $4 n^{\circ} 2$ - Gravure d'ambiance ibère où apparait à droite l'anthroponyme BELSTRATOLO interprété en BELS fils de TARTOLO ou BELSTAR fils de TOLO. Epoque ibère - 200 à 100 av. J.-C.) et (Fig. n 1 : Osséja Zone 2 - Roche $5, \mathrm{n}^{\circ} 56$ - Cavalier à pied menant son cheval par la bride. Fin XIIe s. - début XIIIe s.0)].

De gauche à droite :

- un pentacle,

- deux traits tracés suivant la technique naviforme,

- deux animaux dont un plus petit (peut-être biche et faon ?),

- une frise,

- un anthropomorphe,

- des rouelles et des zigzag.

À l'extrême droite, quatre graffiti en caractères ibèriques superposés, soit dix-sept signes identifiables.

\section{Observation}

Le relevé a été envoyé au professeur Untermann qui fait le commentaire suivant : « Le graffiti Err 2 est un très beau nouvel apport aux mots parfaitement ibériques de la Cerdagne. BELSTARTOLO admet la séparation en deux noms de personnes : ou BELS fils de TARTOLO ou bien BELSTAR fils de TOLO. BO doit être un suffixe».

\section{Date présumée}

Ibérique - 200 à 100 av. J.-C. 


\section{Err-Roc de Carbanet - Roche $2 \mathrm{n}^{\circ} 2$ Description}

Deux étoiles et deux scalariformes, associés aux initiales JO (Fig. $n^{\circ} 1$ : Osséja - Zone 2 Roche $5, n^{\circ} 56$ - Cavalier à pied menant son cheval par la bride. Fin XIIe s. - début XIIIe s.1).

\section{Observation}

JO sont les initiales de Joseph Orriols. C'est le frère du berger Pierre Orriols, comme lui, il était berger et garde-champêtre. Sur d'autres dessins, nous avons son nom en toutes lettres. Il imite ici des gravures anciennes qu'il a pu voir sur la montagne.

\section{Date présumée}

Contemporain, vers 1950.

\section{Err - Els Cortals - Roche $1 \mathrm{n}^{\circ} 1$}

\section{Description}

C'est encore une gravure tracée par le berger Joseph Orriols (Fig. ${ }^{\circ} 1$ : Osséja - Zone 2 Roche $5, n^{\circ} 56$ - Cavalier à pied menant son cheval par la bride. Fin XIIe s. - début XIIIe s.2). À gauche, un sujet anthropomorphe est dessiné suivant une technique bien connue dite « à la 6-4-2 ». La flèche indique la direction du village. Cette flèche est soulignée par un signe en fer à cheval et un cercle coupé d'une croix.

\section{Observation}

Ce n'est pas la première fois que nous trouvons la signature de ce berger dans la montagne d'Err. Il est intéressant de noter qu'il a tenu à s'identifier comme berger. La flèche est tracée ici dans le sens de la marche, il a voulu indiquer au promeneur la direction du village. Les deux signes pourraient bien figurer des marques de berger (fer à marquer) dont un des dessins, trouvé dans une zone proche, représente le même signe.

\section{Date présumée}

1954. 


\section{Err - El Bousquet - Roche $2 \mathrm{n}^{\circ} 1$}

\section{Description}

Une rosace décorée d'une double rangée de festons intérieurs (Fig. $n^{\circ} 1$ : Osséja - Zone 2 Roche $5, n^{\circ} 56$ - Cavalier à pied menant son cheval par la bride. Fin XIIe s. - début XIIIe s.3). Petite rosace au centre, les traits font $0,1 \mathrm{~cm}$ d'épaisseur et $0,04 \mathrm{~cm}$ de profondeur. Les traits de la rosace intérieure sont plus fins. À droite, un cercle tracé suivant la même technique. À l'intérieur la date 1899, entre les deux dessins (en haut) tracé au couteau les initiales PO (Pierre Oriol sûrement). Entre les cercles (en bas), les initiales PR tracées suivant la technique de gravures des cercles.

\section{Observation}

Il est difficile d'attribuer le dessin à Pierre Oriol, les traits de PR sont identiques à ceux des cercles. Si nous avons pu identifier le nom qui correspond aux initiales PO (Pierre Oriol, berger à Err), en revanche PR nous est inconnu. L'attribution du dessin à l'un ou l'autre reste une énigme. On peut simplement dire que PR est tracé suivant la technique de la rosace, par contre la date de 1899 se rattache plus à Pierre Oriol qui en 1899 avait 23 ans.

\section{Période présumée}

Époque contemporaine (1899).

\section{Latour de Carol}

\section{Latour de Carol 2 - Roche 9}

\section{Description}

Groupe de trois guerriers en armes (Fig. $n^{\circ} 1$ : Osséja - Zone 2 - Roche $5, n^{\circ} 56$ - Cavalier à pied menant son cheval par la bride. Fin XIIe s. - début XIIIe s.7). Les trois sujets sont, à quelques détails près, traités de la même façon. Le dessin s'articule autour d'une croix où l'axe vertical représente le corps et l'axe horizontal, les bras. La tête, fixée à l'extrémité supérieure de l'axe, est coiffée d'un casque pointu. La technique de traçage est linéaire.

Sur le sujet de gauche, la tête n'est pas dessinée, seul le casque est figuré. L'épaisseur du corps est obtenue par une ligne qui, partant au niveau des jambes, recoupe l'axe de la croix par un arc de cercle pour revenir vers le bas où il termine l'autre jambe. Ce modèle s'applique aux deux autres dessins supérieurs.

Celui du bas est différent. Le corps est traité à l'identique sauf que les lignes se referment au niveau du bassin. Les pieds sont pectiniformes.

Le milieu du corps des trois sujets est dessiné sous la forme d'un vague quadrilatère croisillonné et nous l'interprétons comme un haut-de-chausse. Le sujet central tient dans 
sa main droite un objet qui peut être interprété comme un arc ou une arbalète très mal dessinée, ou comme un oriflamme. Le sujet du bas tient dans sa main droite une lance et un objet indéterminé dans sa main gauche, peut-être une deuxième lance. On notera que les mains ont une forme ramifiée filiforme. Au centre du dessin, huit traits parallèles complètent cette scène.

\section{Observation}

La tenue vestimentaire de ces trois guerriers a une grande importance pour la chronologie. Le casque pointu, nous l'avons vu lors de l'étude du cavalier à la lance d'Osséja-Genèvri - Zone 1, est daté des XII ${ }^{\mathrm{e}}$ s. et XIII ${ }^{\mathrm{e}}$ s., alors que la chronologie du hautde-chausse est plus précise puisque ce vêtement, dérivant des braies, n'et connu qu'à la fin $\mathrm{du} X \mathrm{XV}^{\mathrm{e}} \mathrm{s}$.

\section{Date présumée}

Fin du XV s.

\section{Latour de Carol 2 - Roche 19}

\section{Description}

Graffite ibère associé à un graffite latin [ (Fig. $\mathrm{n}^{\circ} 1$ : Osséja - Zone 2 - Roche $5, \mathrm{n}^{\circ} 56$ Cavalier à pied menant son cheval par la bride. Fin XIIe s. - début XIIIe s.4) et (Fig. $\mathrm{n}^{\circ} 1$ : Osséja - Zone 2 - Roche $5, \mathrm{n}^{\circ} 56$ - Cavalier à pied menant son cheval par la bride. Fin XIIe s. - début XIIIe s.5)].

\section{Observation}

Selon le professeur Untermann, ils sont de la même main. Si on considère la lenteur avec laquelle a dû se répandre le latin en Cerdagne, on ne peut raisonnablement dater cette inscription qu'antérieurement au $\mathrm{I}^{\mathrm{er}} \mathrm{s}$. av. J.-C. Cette hypothèse paraît confirmée par la découverte récente d'un phénomène identique à Cogul en Espagne, près de Lérida. Il s'agit de l'association d'une inscription ibérique et d'un texte latin qui ne sont autres que deux vers, extraits des Géorgiques de Virgile. L'auteur a vécu de 70 à $19 \mathrm{av}$. J.-C., le poème fût publié entre 39 et 29 av. J.-C. : la gravure ne saurait être antérieure et serait donc datable de la fin du $\mathrm{I}^{\mathrm{er}} \mathrm{s}$. avant notre ère ou postérieurement.

\section{Date présumée}

Postérieur à la deuxième moitié du $\mathrm{I}^{\mathrm{er}} \mathrm{s}$. avant J.-C. 


\section{Latour de Carol 2 - Roche 10}

\section{Description}

Gravure extrêmement complexe et surchargée, avec de très nombreux symboles. Le dessin peut se décomposer en deux parties (Fig. $\mathrm{n}^{\circ} 1$ : Osséja - Zone 2 - Roche $5, \mathrm{n}^{\circ} 56$ Cavalier à pied menant son cheval par la bride. Fin XIIe s. - début XIIIe s.6). Dans la partie haute, les zigzags dominent. La partie basse, plus dépouillée, est surtout composée de lignes linéaires. En haut et à gauche, signe en forme de $\mathrm{X}$ avec une ligne droite reliant la partie supérieure des deux axes. À gauche, au centre des zigzags, on distingue très nettement un anthropomorphe en forme de croix. À l'extrémité inférieure de l'axe vertical, deux lignes obliques dirigées vers le bas figurent les jambes terminées par des extrémités ramifiées filiformes. L'axe horizontal légèrement oblique représente les bras. Ses extrémités finissent en mains ramifiées. Un signe arbalétiforme semble être tenu en main côté droit; ce signe se compose d'un arc positif et d'un axe vertical terminé par une fourche. À gauche, un losange avec diagonales est dessiné sous le bras. Cette composition primitive est oblitérée par au moins vingt signes en forme de zigzag. Dessous, des lignes obliques et horizontales recoupent trois longues lignes verticales.

\section{Observation}

Il ressort de cette figure une grande force symbolique. La puissance de l'anthropomorphe armé est accentuée ou peut-être réduite par la force des éclairs. On peut imaginer que le dessinateur, qui est le narrateur, ait voulu détruire par la foudre donc par le feu, la puissance du sujet armé. A contrario, il a très bien pu vouloir augmenter cette puissance, en lui donnant par la foudre, qui est aussi synonyme de pluie, un sens fécondateur. Ces observations restent toutefois une simple vue de l'esprit qui sont certainement à cent lieux de la réalité voulue par l'auteur de la gravure.

\section{Date présumée}

Indéterminée.

\section{ANNEXES}


Fig. $\mathrm{n}^{\circ} 1$ : Osséja - Zone 2 - Roche $5, \mathrm{n}^{\circ} 56$ - Cavalier à pied menant son cheval par la bride. Fin XIle s. - début XIIle s.
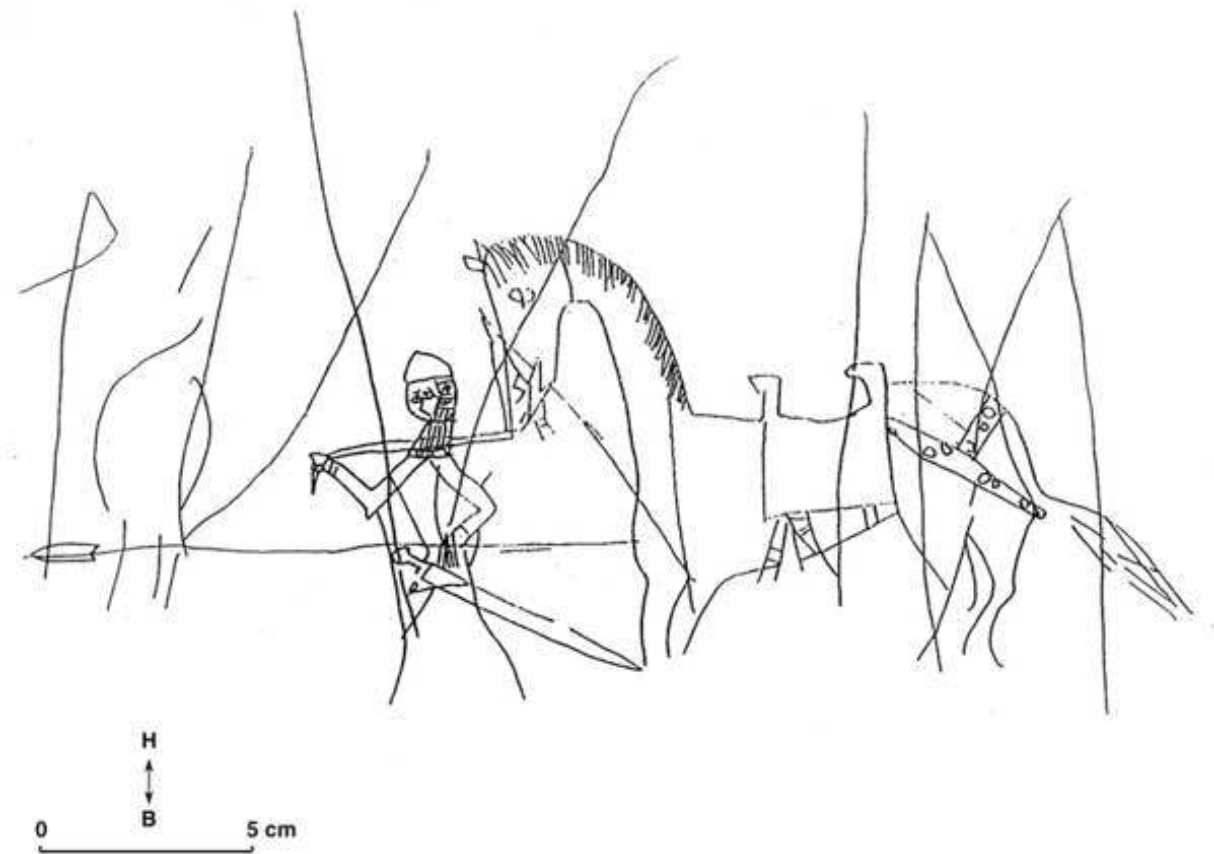

Auteur(s) : Campmajo, Pierre. Crédits : ADLFI - Campmajo, Pierre (2004)

Fig. $n^{\circ} 2$ : Osséja - Zone 2 - Roche $5, n^{\circ} 56$ - Détail du cavalier à pied menant son cheval par la bride. Fin XIle s. - début XIIle s.

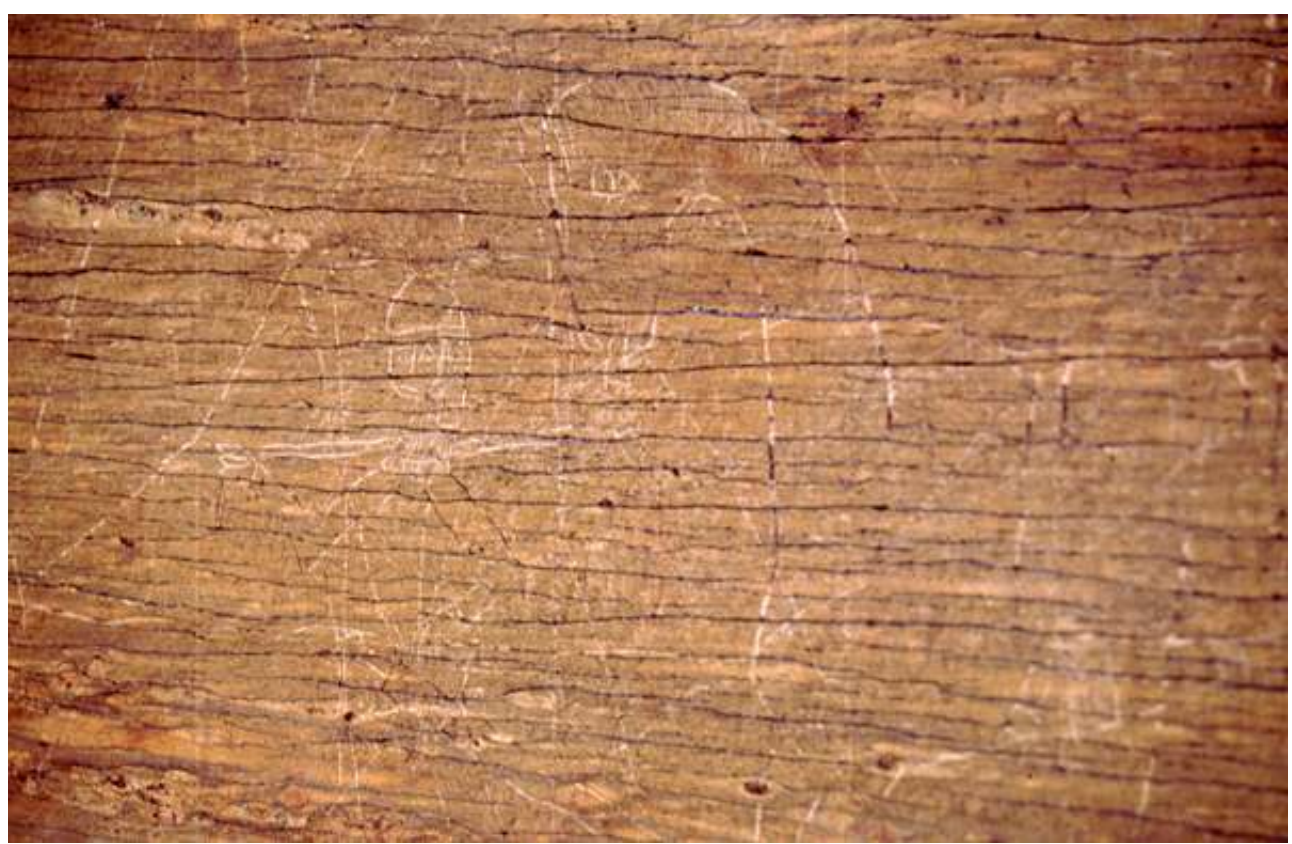

Auteur(s) : Campmajo, Pierre. Crédits : ADLFI - Campmajo, Pierre (2004) 
Fig. $n^{\circ} 3$ : Osséja - Zone 2 - Roche $1, n^{\circ} 4$ - Groupe de chasseurs ou de guerriers en armes. Époque médiévale

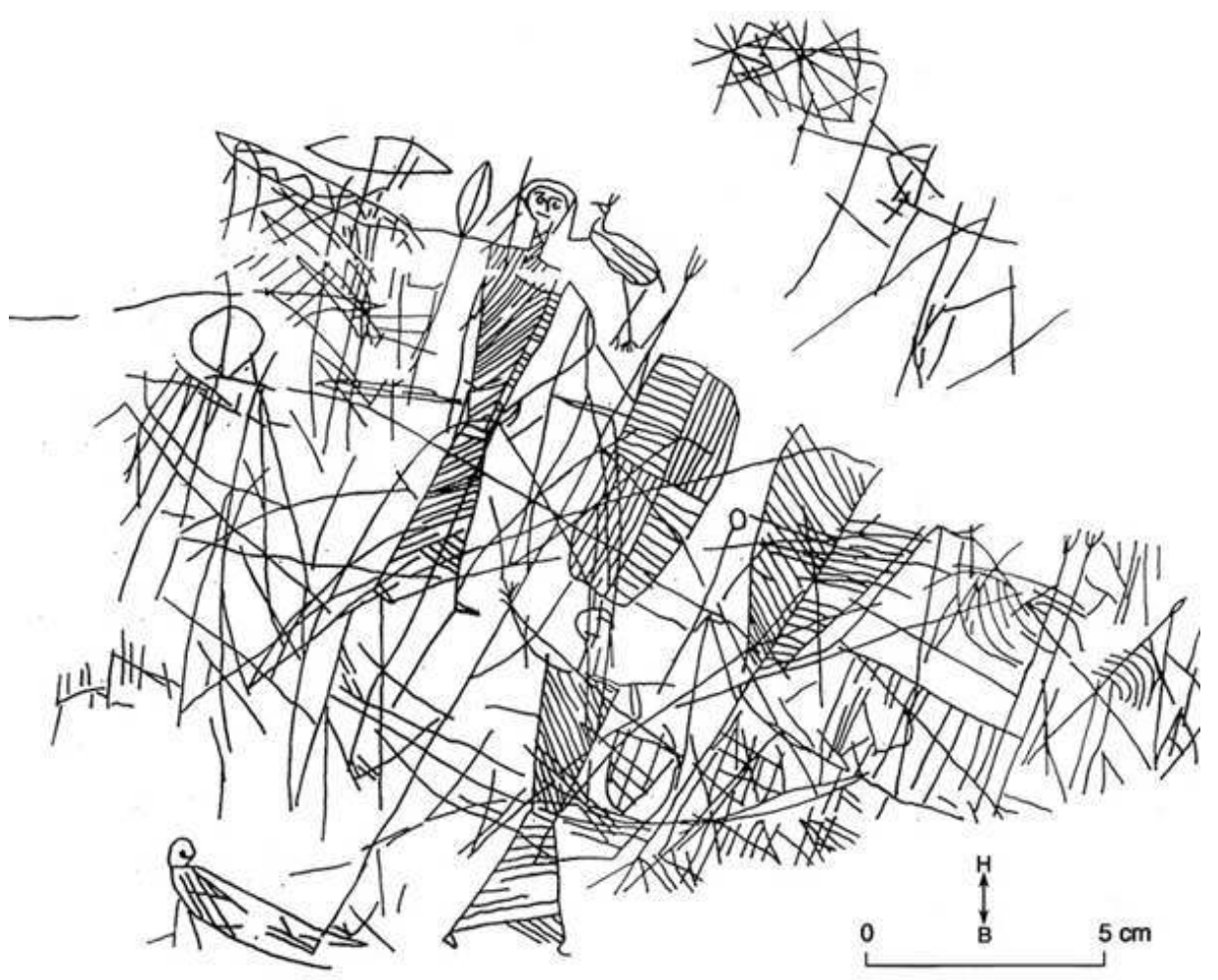

Auteur(s) : Campmajo, Pierre. Crédits : ADLFI - Campmajo, Pierre (2004) 
Fig. $n^{\circ} 4$ : Osséja - Zone 2 - Roche $1 n^{\circ} 4$ - Détail du panneau représentant un sujet anthropomorphe en arme, surmonté d'un oiseau

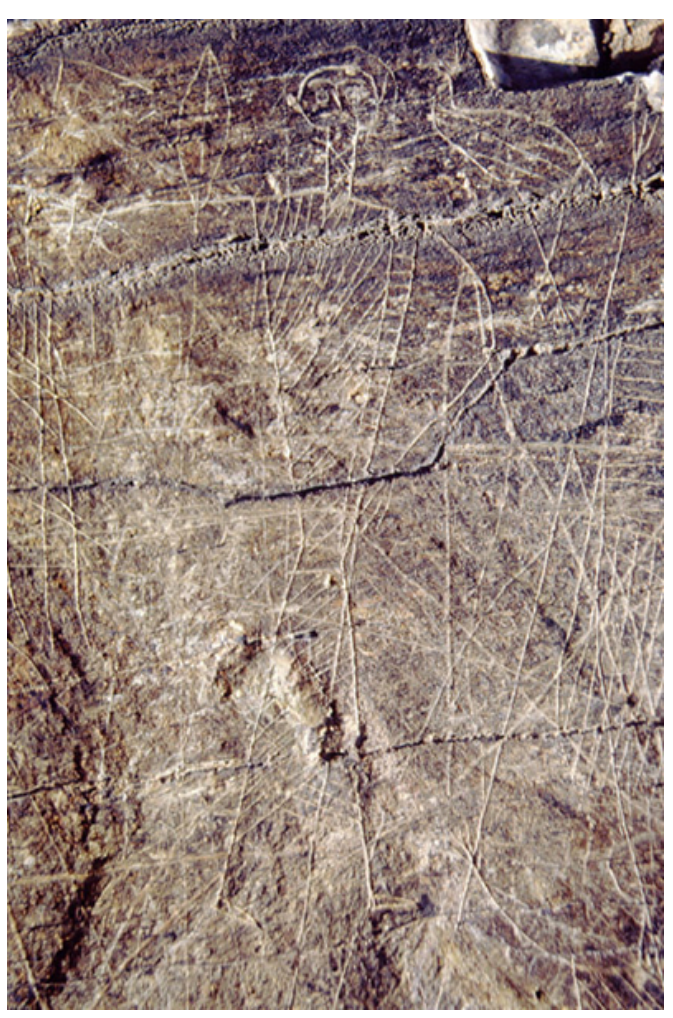

Auteur(s) : Campmajo, Pierre. Crédits : ADLFI - Campmajo, Pierre (2004) 
Fig. $n^{\circ} 5$ : Osséja-Corbera - Zone 6 - Roche $2 n^{\circ} 1$ - Quatre lignes d'écriture ibère. Époque ibère 200 à 100 av. J.-C.

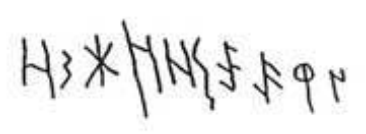

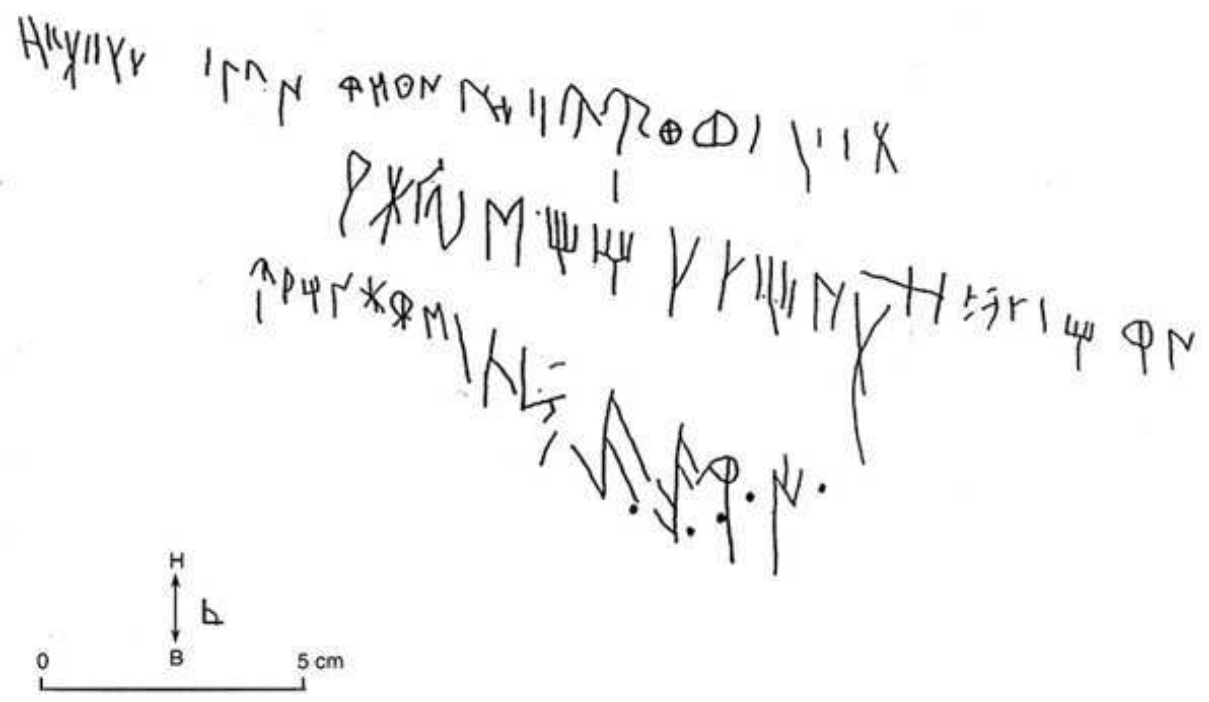

Auteur(s) : Campmajo, Pierre. Crédits : ADLFI - Campmajo, Pierre (2004) 
Fig. $n^{\circ} 6$ : Osséja-Corbera - Zone 6 - Roche $1 n^{\circ} 1$ - Ecriture ibère. Époque ibère - 200 à 100 avant J.-C.
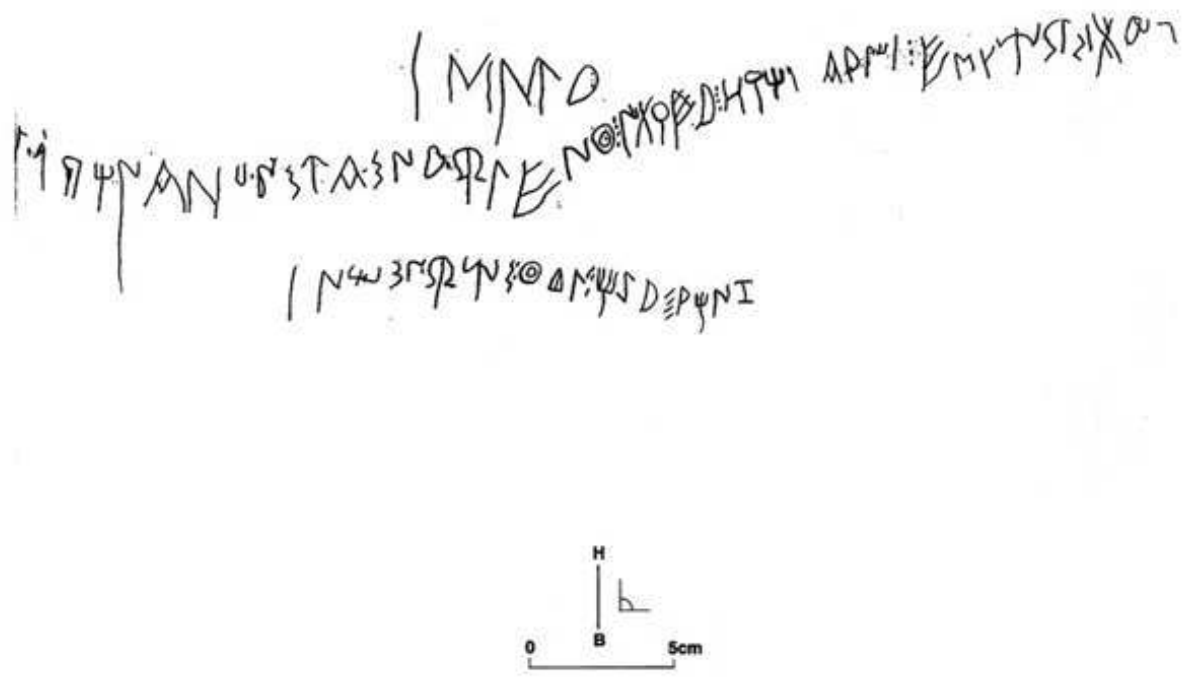

Auteur(s) : Campmajo, Pierre. Crédits : ADLFI - Campmajo, Pierre (2004) 
Fig. $n^{\circ} 7$ : Osséja-Carrière de la Cabanette - Roche $1 n^{\circ} 1$ - Guerrier armé associé à un faisceau de lances. Moyen Âge, sous réserve
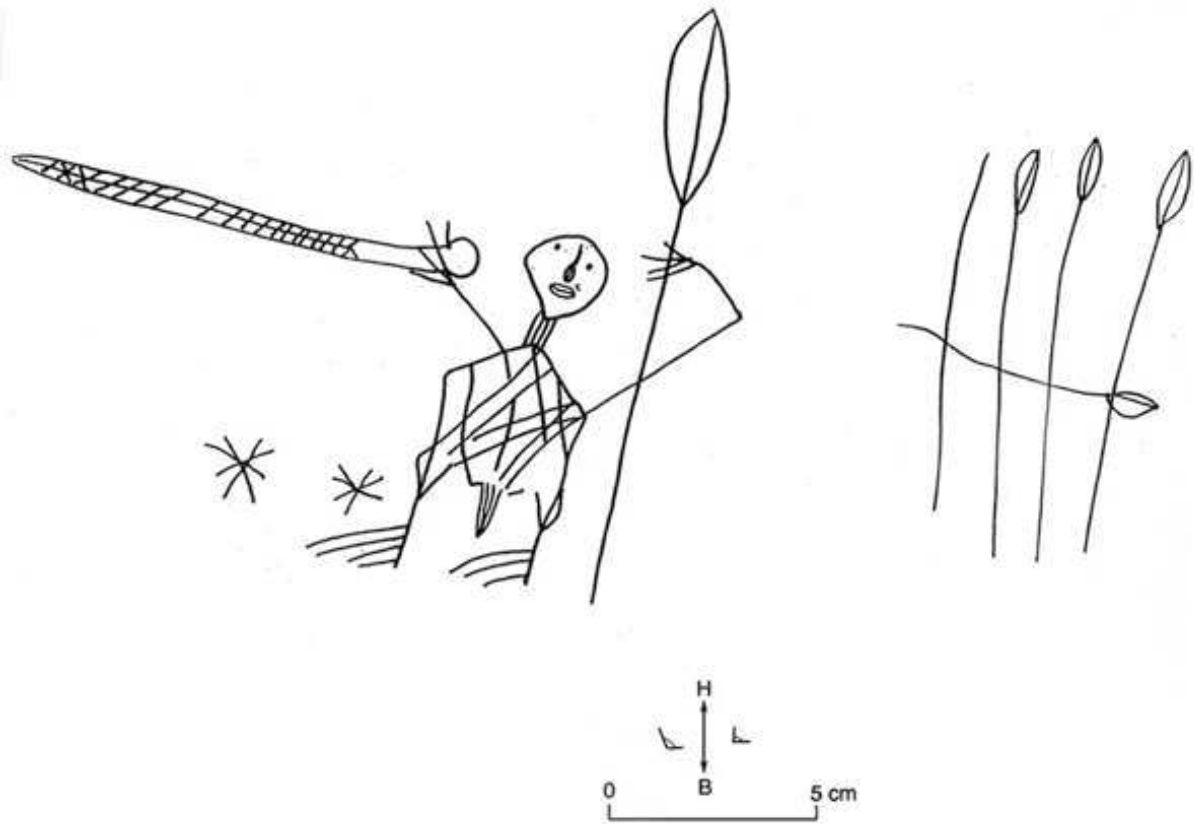

Auteur(s) : Campmajo, Pierre. Crédits : ADLFI - Campmajo, Pierre (2004) 
Fig. $\mathrm{n}^{\circ} 8$ : Osséja - Zone 2 - Roche 4 n$^{\circ} 19$ - Groupe d'anthropomorphes aux formes diverses. Époque ibère

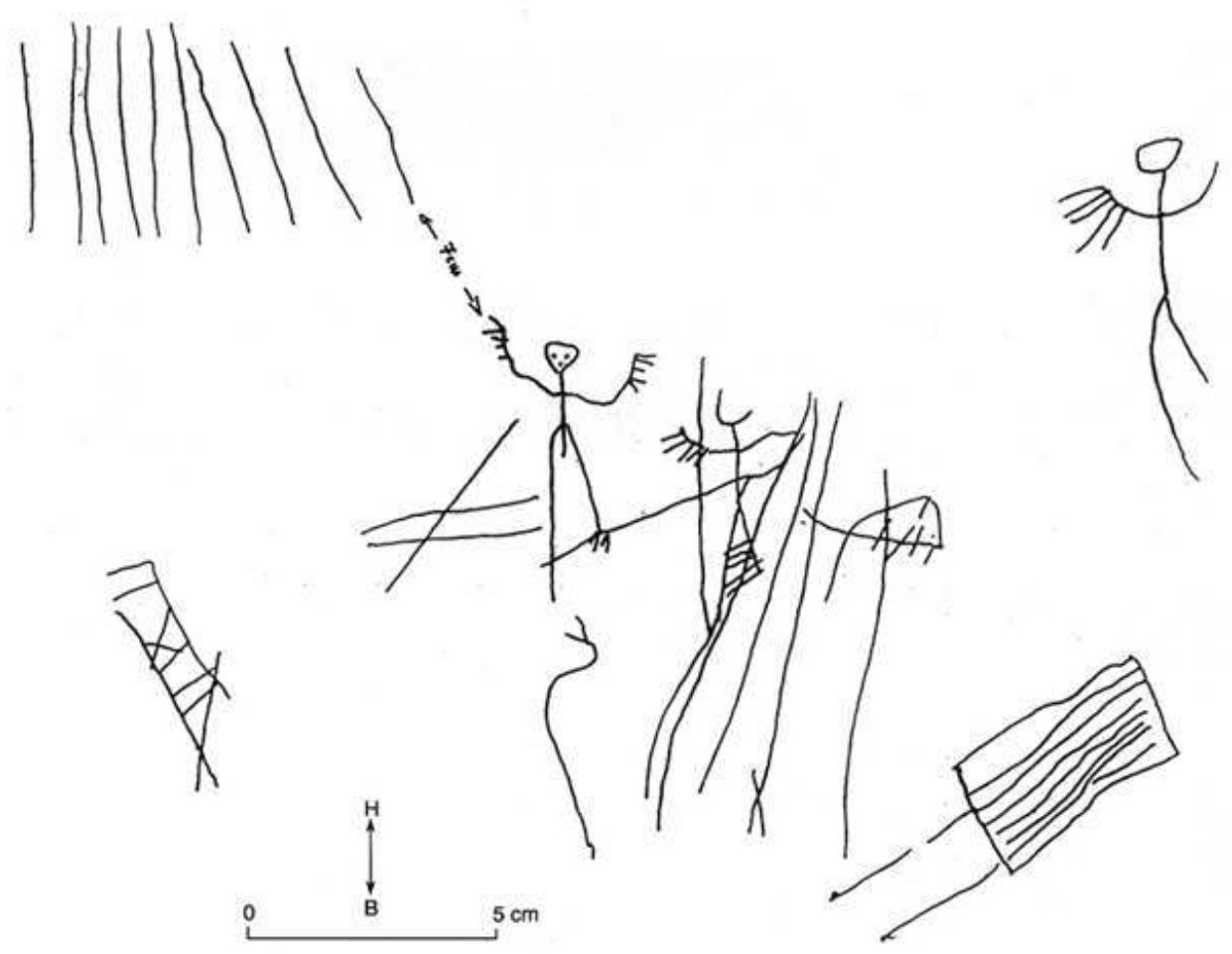

Auteur(s) : Campmajo, Pierre. Crédits : ADLFI - Campmajo, Pierre (2004) 
Fig. $n^{\circ} 9$ : Err-Carbanet - Roche $4 n^{\circ} 2$ - Gravure d'ambiance ibère où apparaît à droite l'anthroponyme BELSTRATOLO interprété en BELS fils de TARTOLO ou BELSTAR fils de TOLO. Epoque ibère -200 à 100 av. J.-C.

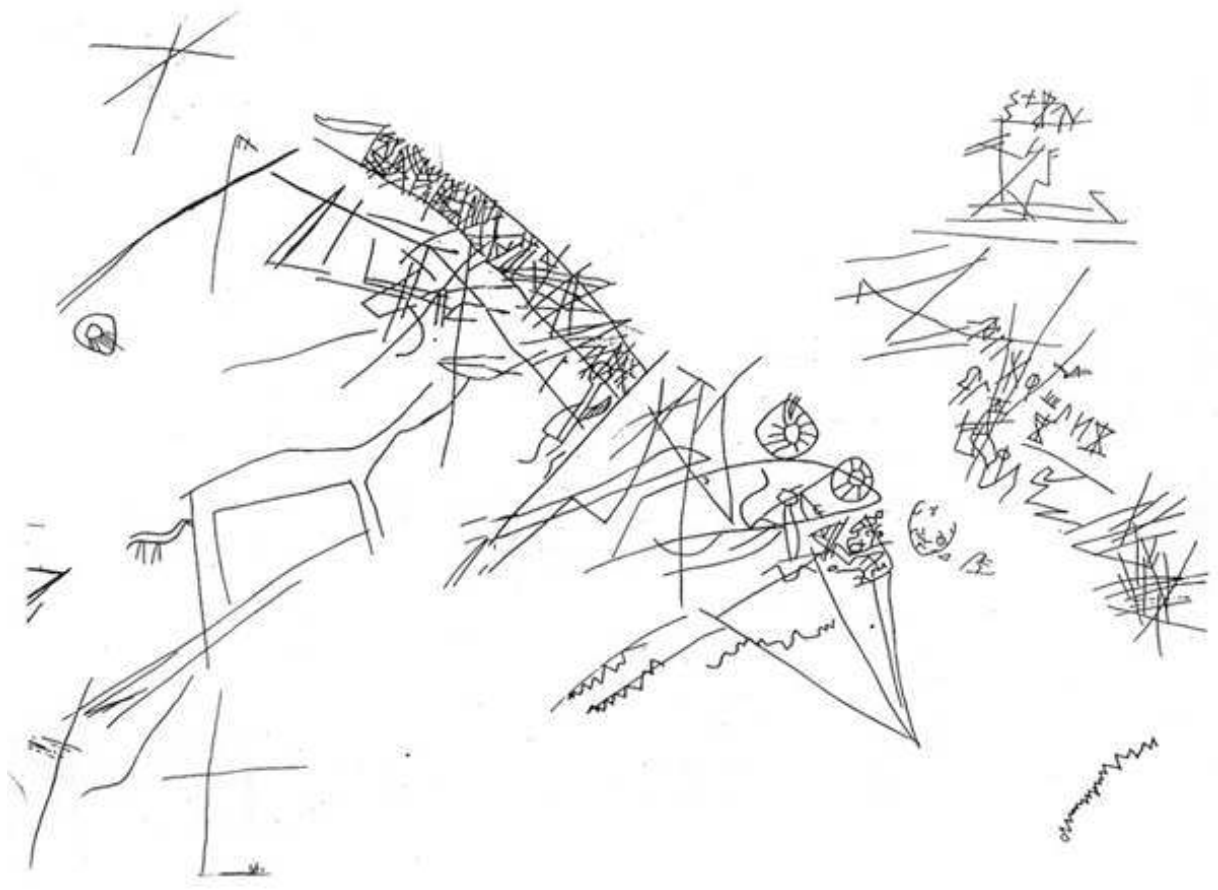

Auteur(s) : Campmajo, Pierre. Crédits : ADLFI - Campmajo, Pierre (2004)

Fig. $n^{\circ} 10$ : Err-Carbanet - Roche $4 n^{\circ} 2$ - Détail de la gravure ibère fig. 9

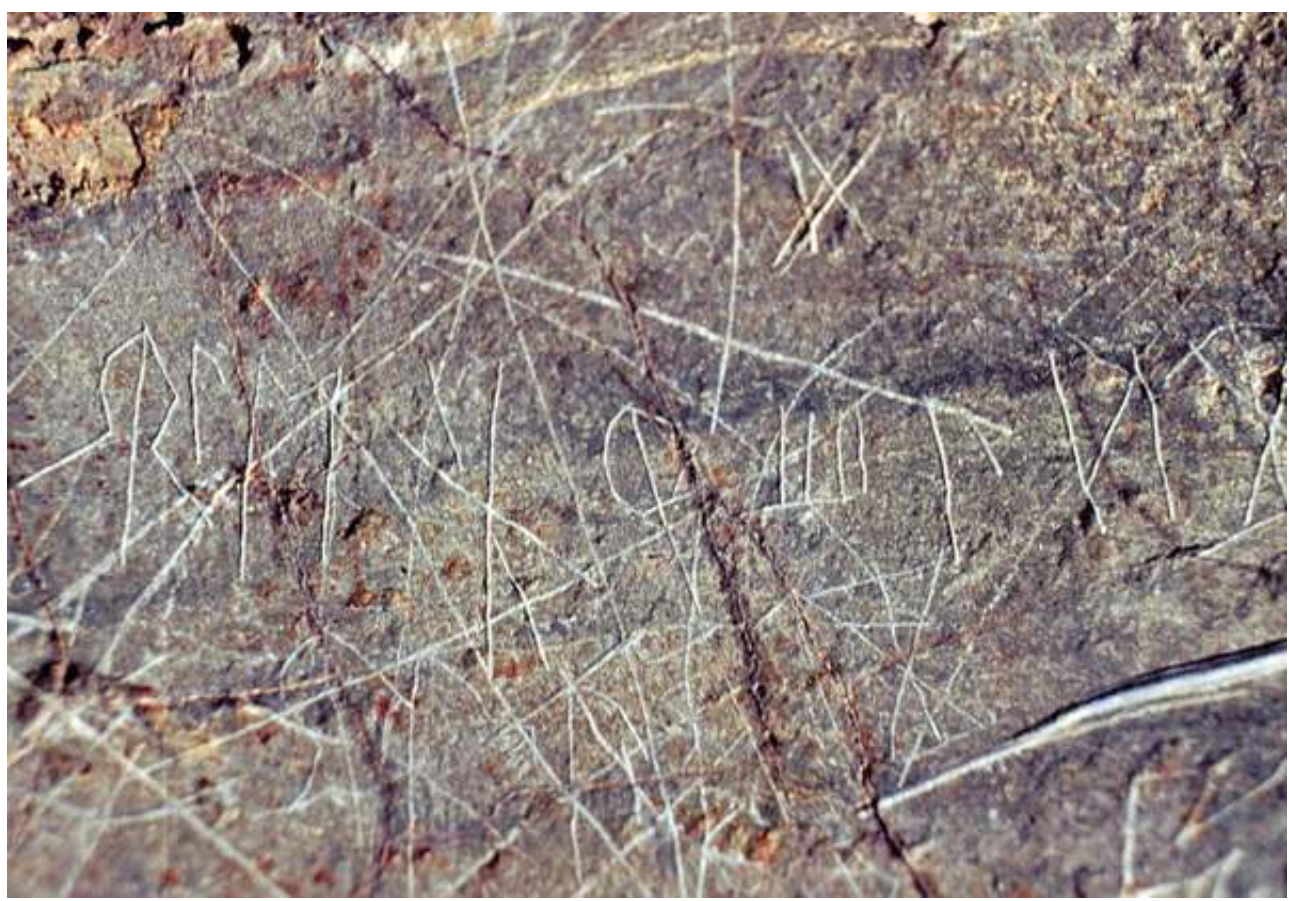

Auteur(s) : Campmajo, Pierre. Crédits : ADLFI - Campmajo, Pierre (2004) 
Fig. $\mathrm{n}^{\circ} 11$ : Err-Carbanet - Roche $2 \mathrm{n}^{\circ} 2$ - Gravure contemporaine copiant des gravures plus anciennes. Initiales JO de Joseph Orriols, berger

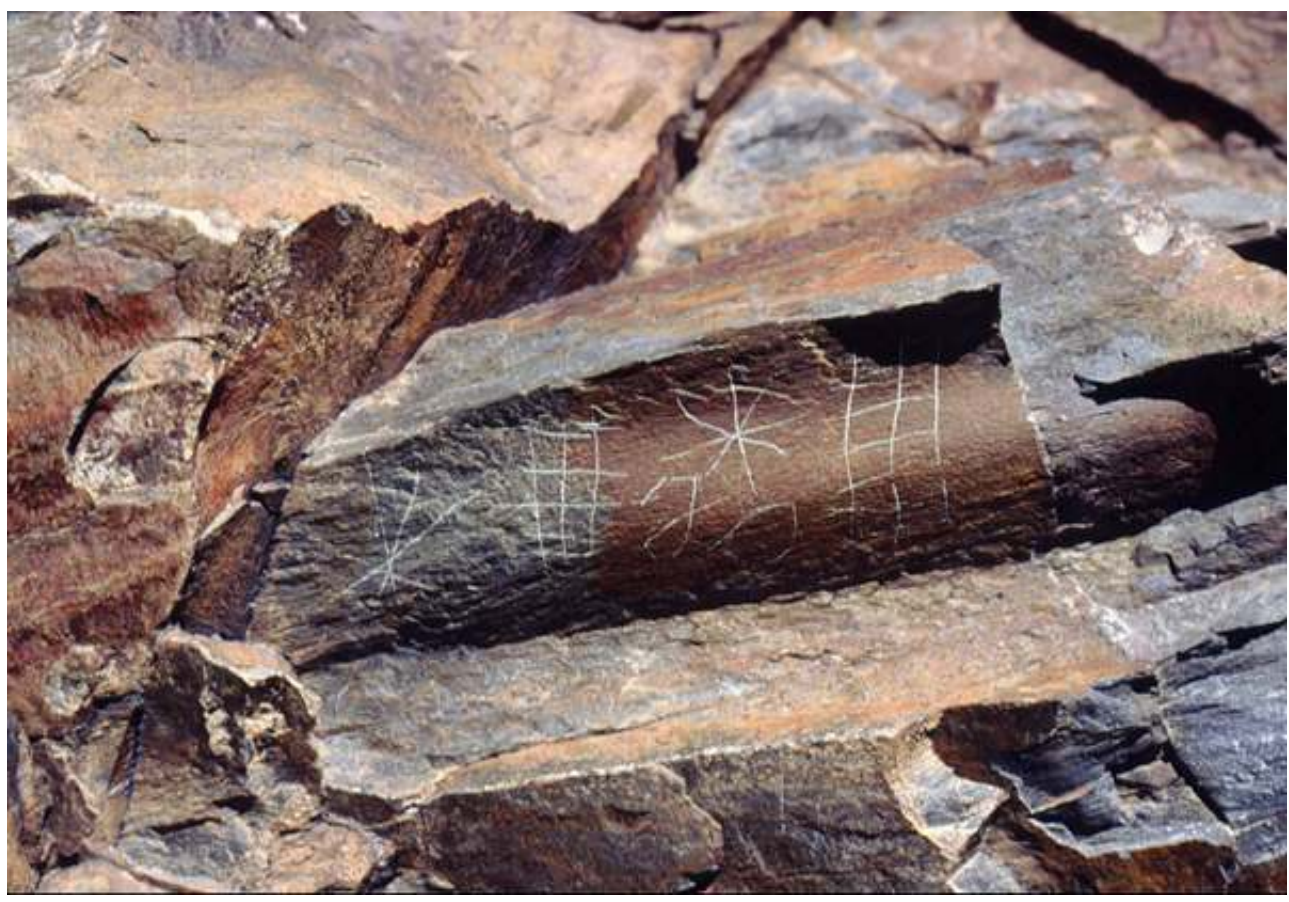

Auteur(s) : Campmajo, Pierre. Crédits : ADLFI - Campmajo, Pierre (2004)

Fig. $n^{\circ} 12$ : Err-Els Cortals - Roche $1 n^{\circ} 1$ - Sujet anthropomorphe avec une flèche, soulignée par un signe en fer à cheval et un cercle coupé d'une croix. Contemporain : 1954
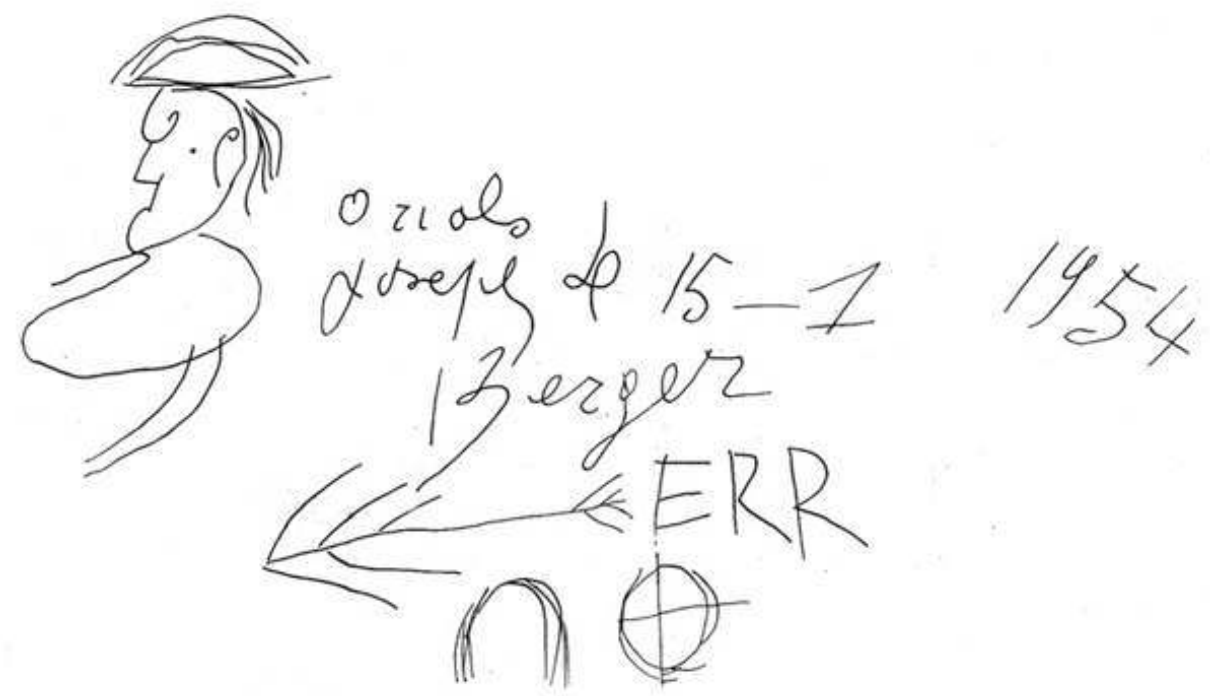

Auteur(s) : Campmajo, Pierre. Crédits : ADLFI - Campmajo, Pierre (2004) 
Fig. $n^{\circ} 13$ : Err-El Bosquet - Roche $2 n^{\circ} 1$ - Gravure contemporaine (rosace) attribuée au berger Pierre Orriols. Contemporain : 1899

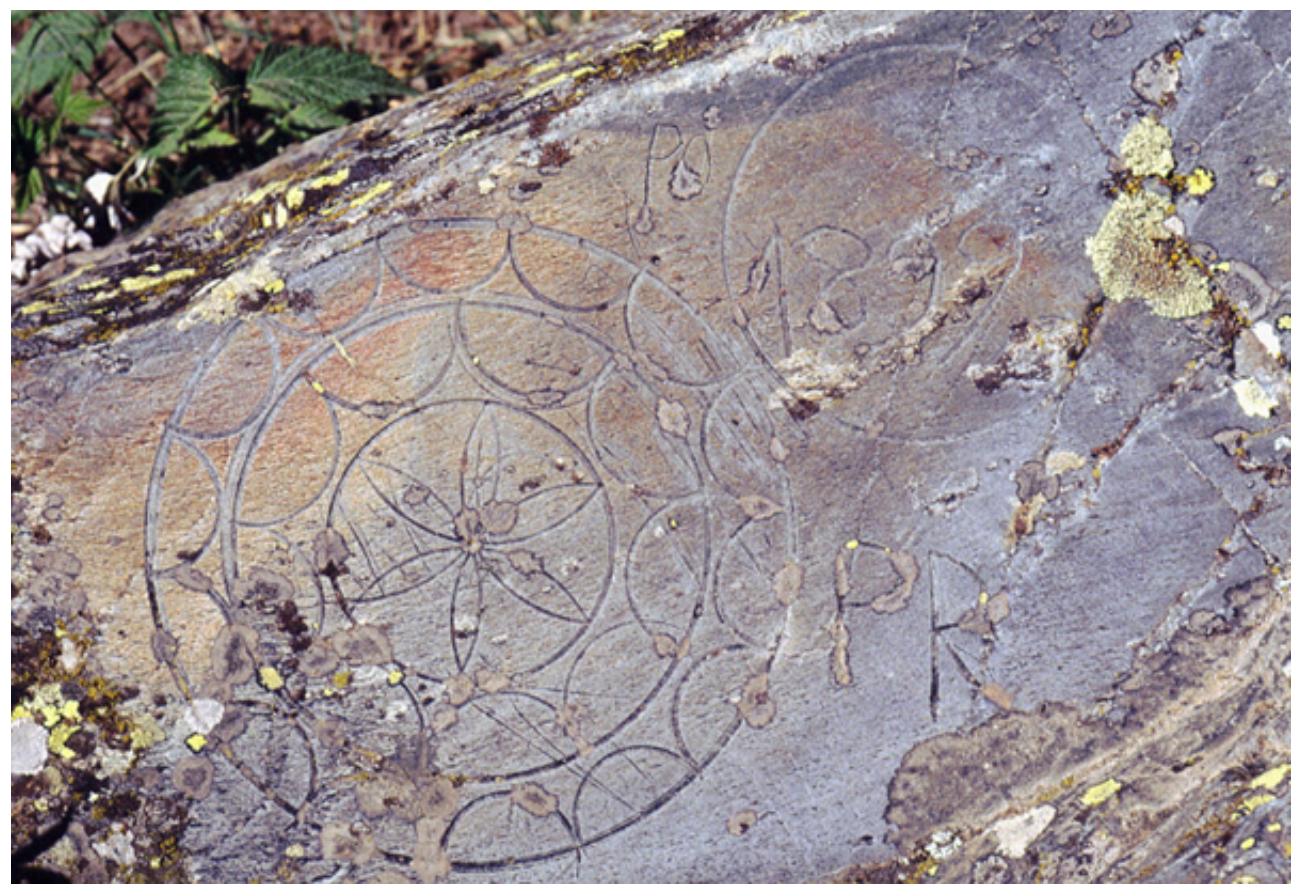

Auteur(s) : Campmajo, Pierre. Crédits : ADLFI - Campmajo, Pierre (2004)

Fig. ${ }^{\circ} 14$ : Latour de Carol 2 - Roche $n^{\circ} 19$ - Graffiti ibère associé à un graffiti latin. Deuxième moitié du 1er s. av. J.-C.

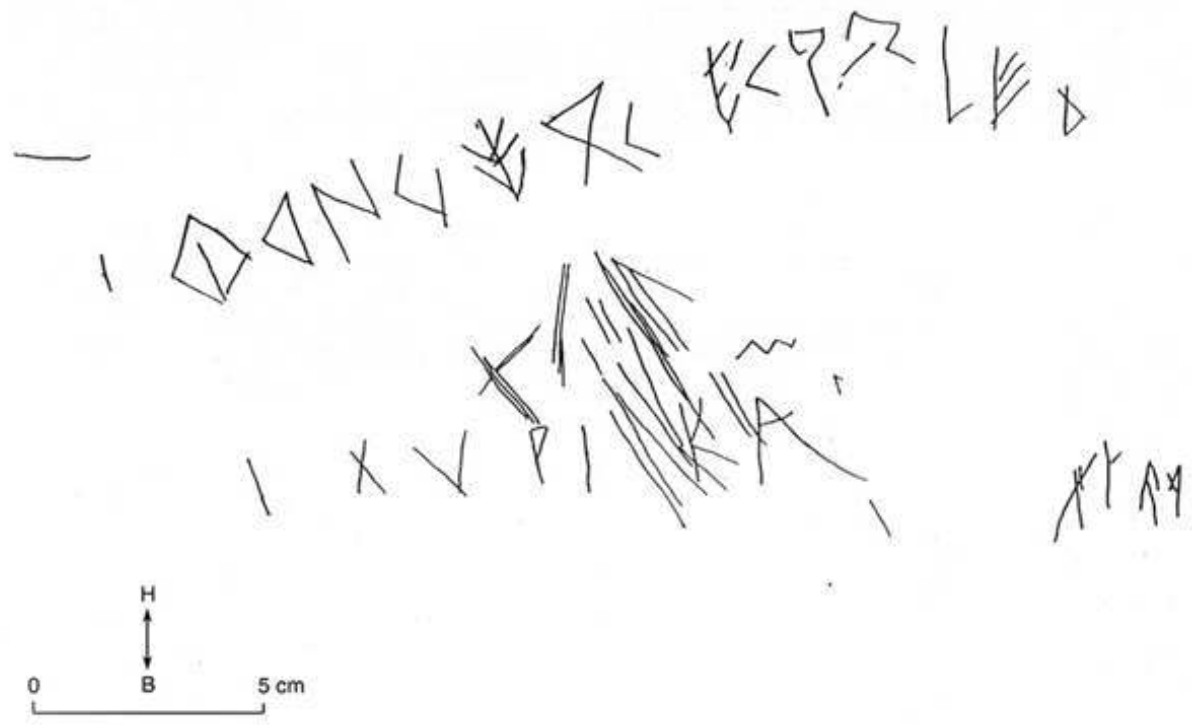

Auteur(s) : Campmajo, Pierre. Crédits : ADLFI - Campmajo, Pierre (2004) 
Fig. $n^{\circ} 15$ : Latour de Carol 2 - Roche $n^{\circ} 19$ - Graffiti ibère associé à un graffiti latin. Deuxième moitié du 1er s. av. J.-C.

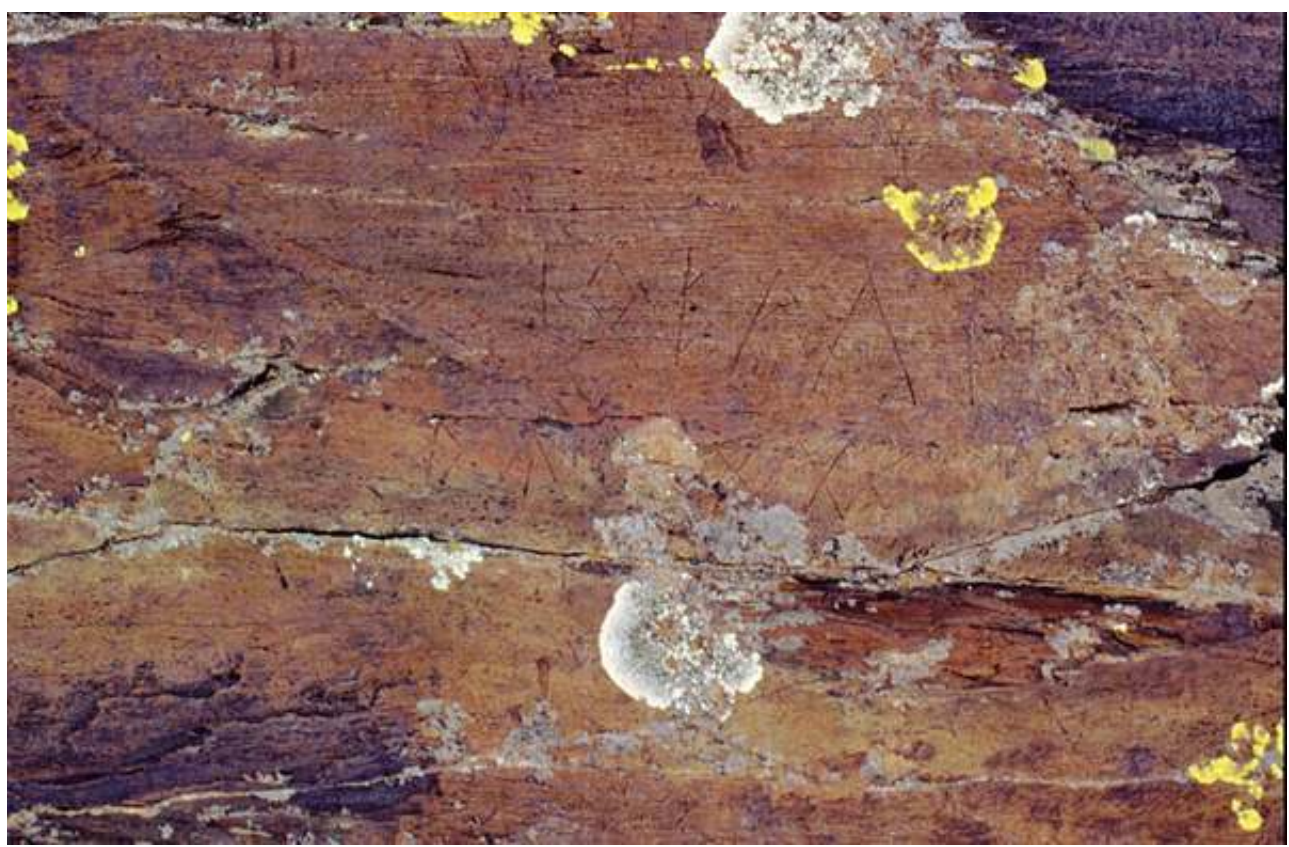

Auteur(s) : Campmajo, Pierre. Crédits : ADLFI - Campmajo, Pierre (2004)

Fig. $n^{\circ} 16$ : Latour de Carol 2 - Roche $n^{\circ} 10$ - Gravure complexe avec au centre, une figure anthropomorphe oblitérée par de nombreux zigzags. Époque ibère ou médiévale, sans plus de précision
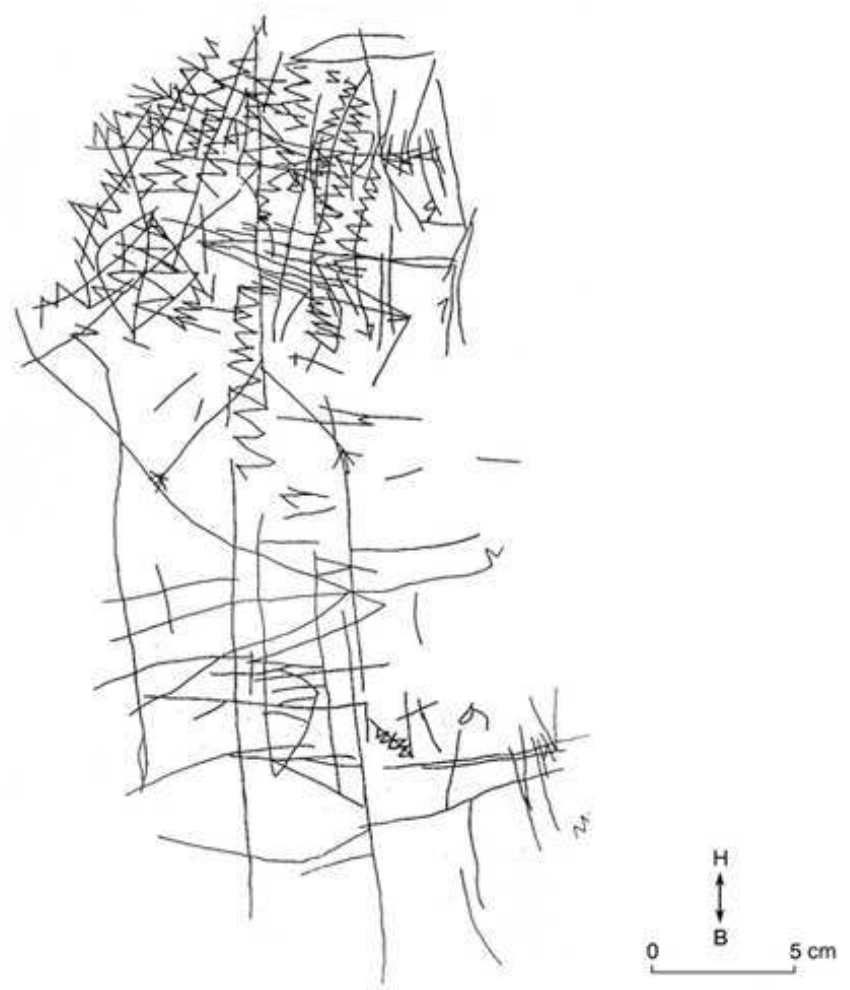

Auteur(s) : Campmajo, Pierre. Crédits : ADLFI - Campmajo, Pierre (2004) 
Fig. $\mathrm{n}^{\circ} 17$ : Latour de Carol 2 - Roche $\mathrm{n}^{\circ} 9$ - Groupe de trois guerriers en armes portant casque et haut-de-chausse. Moyen Âge : fin XVe s.

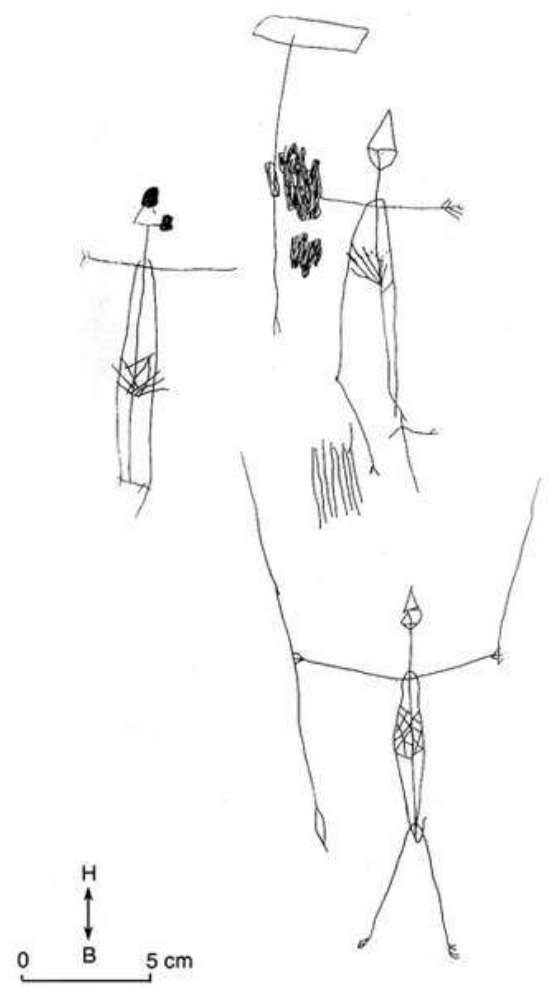

Auteur(s) : Campmajo, Pierre. Crédits : ADLFI - Campmajo, Pierre (2004) 
Fig. $\mathrm{n}^{\circ} 18$ : Osséja - Zone 1 - Roche $3 \mathrm{n}^{\circ} 1$ - Scène composée d'éléments figuratifs, anthropomorphe, spirale et zigzag. Moyen Âge

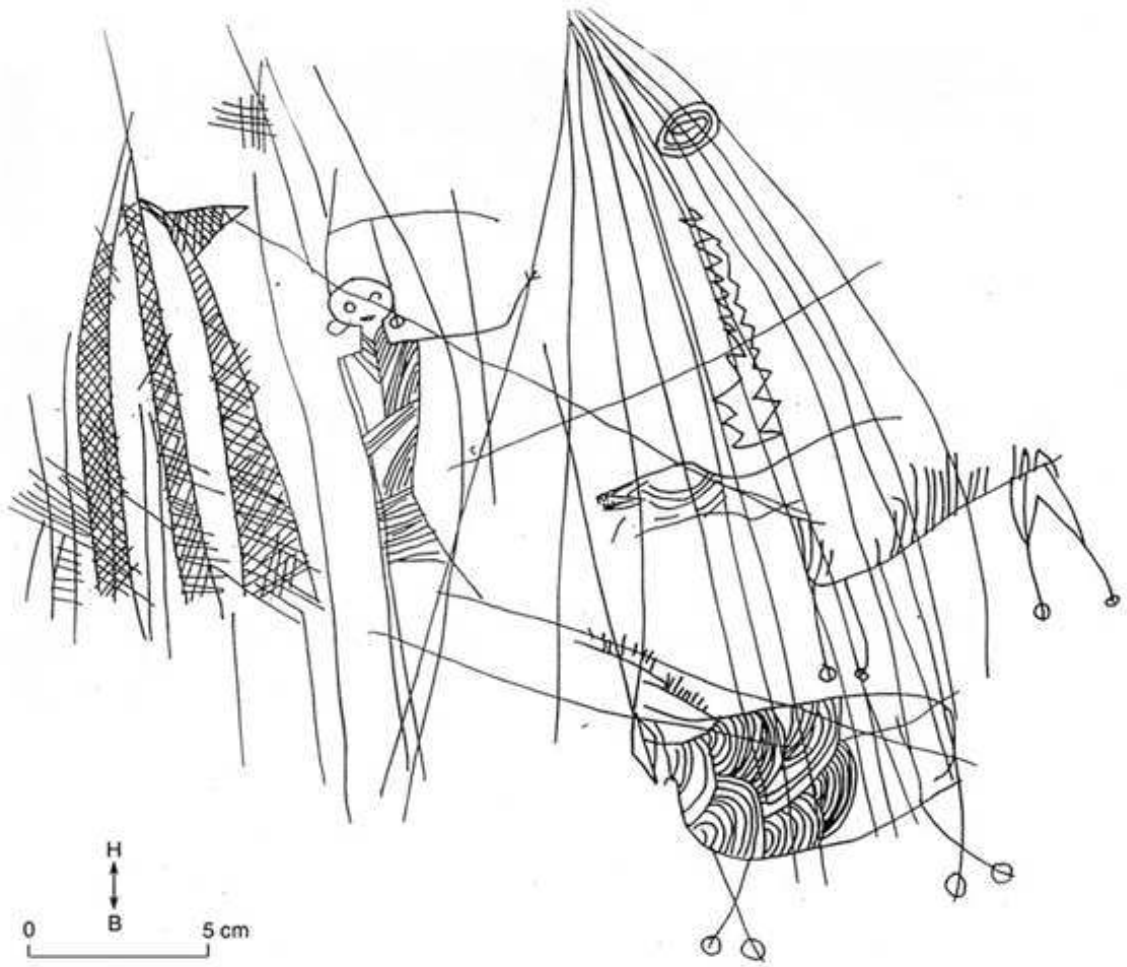

Auteur(s) : Campmajo, Pierre. Crédits : ADLFI - Campmajo, Pierre (2004)

Fig. ${ }^{\circ} 19$ : Osséja - Corbera Roche Rendut - Roche $1 n^{\circ} 1$

La première ligne du texte, placée à peu près au-dessus du milieu des deux autres, est composée de six lettres dont on n'identifie que les dernières quatre lettres avec certitude. Hauteur: $1,5 \mathrm{~cm}$ à $3 \mathrm{~cm}$.

básbanir ou básbalir

[On ne peur affirmer que l'on ait à faire à une ligature $(n+1)$ ou bien à deux lettres séparées (li)] Cp., avec'r au lieu de r, ]śsbanir sur un vase peint de Liria (F.13.47), et avec suffixe -(e)ke au lieu de -ir, baśbaneke sur le plomb III de Pech-Maho.

Les deux dernières lignes sont légèrement ondulées:

hauteur des lettres: $0,8 \mathrm{~cm}$ à $2 \mathrm{~cm}$;

séparation des mots par interponction qui se compose de deux, trois ou quatre traits verticaux.

Ortinkalibisubasir : belenku : itafer : ortinkalibie-sambe : borti

$$
\begin{array}{llllllll}
5 & 10 & 15 & 20 & 25 & 30 & 35 & 40
\end{array}
$$

anbelsibems : kutun : titkirsatin

$5 \quad 10 \quad 15$

L'écriture montre des correspondanes remarquables avec le type graphique employé par les graffiti trouvé à Ullastret (C.2.9 et suiv.) et par le texte dur plom provenant de l'alamós (C.4.1). Jusqu'à la découverte de notre inscription, le signe $\uparrow$ n'étair connu que dans le document de l'alamós. Sauf quelques témoignages isolés et incertains la lettre $\uparrow=m$ n'est employée qu'à Ullastret, Palamós et par les inscriptions peintes sur la céramique de Liria.

Auteur(s) : Campmajo, Pierre. Crédits : ADLFI - Campmajo, Pierre (2004) 
INDEX

Index chronologique : Antiquité tardive, ép. contemporaine, Moyen Âge, Temps Modernes operation Prospection inventaire (PI)

Index géographique : Languedoc-Roussillon, Pyrénées-Orientales (66), Palau-de-Cerdagne peuple Champs d'Urnes, Ibères

anthroponyme Caton l'Ancien 